\title{
Summary of General Discussion and Conclusions
}

FV Zohoori ${ }^{1}$

${ }^{1}$ School of Health \& Social Care, Teesside University, UK.

Key words: Caries, Dental public health, Fluoride(s), Nutrition/nutritional Sciences, Prevention

\section{Corresponding Author:}

Professor Vida Zohoori,

Health and Social Care Institute

Teesside University

Middlesbrough, TS1 3BA

Email:v.zohoori@tees.ac.uk

Tel: $+44(0) 1642342973$

Total number of words: $\quad 1990$

Number of references: $\quad 6$

Number of tables and figures: 0 
The discussion period lasted 40 minutes with questions and comments from those attending the symposium. This was aided by a series of questions provided by the chair which were considered by the panel of the three reviewers (Drs. Spencer, Buzalaf and Mejàre), the two discussants (Drs. Martinez Mier and Walls), as well as by those attending. Comments made, relevant to each question, were recorded by hand and have been summarised. The aim was, first, to see if a consensus could be reached on a choice of valid guidelines for fluoride intake and, second, to identify research objectives for the future.

\section{Question 1: Is there a need for a Dietary Reference Value (DRV) for fluoride?}

At this point, it was clarified that 'DRV' is the terminology used in the UK to refer to 'a series of estimates of the amount of energy and nutrients needed by different groups of healthy people'; whereas it is termed 'Nutrient Reference Value (NRV)' in Australia and 'Recommended Dietary Allowance (RDA)' in the USA.

A comment was made that fluoride is not generally considered as an essential element. However, the point was made that fluoride is believed to be an important and beneficial element due to its ability to protect against dental caries (i.e. pathological demineralisation of calcified tissues) and not dismissing the possibility of fluoride being an essential nutrient. Moreover, it was noted that fluoride has been included in the list of 'ultratrace elements', a term which has arrived in the nutritional literature only since 1980 (Nielsen 1996). Ultratrace elements have been defined as “.... elements with an established, estimated or suspected requirement generally indicated by $\mu \mathrm{g} /$ day. although differing dietary guidance is appropriate for these elements, most need increased attention in future editions of the Recommended Dietary Allowances (RDAs) ....." (Nielson 1996).

The panel agreed on the need for setting DRVs for fluoride to provide a guideline on appropriate levels of fluoride intake. The panel also suggested not using the term 'optimum' but proposed 'appropriate outcomes' when defining the value; i.e. 'a value that provides appropriate outcomes in terms of caries prevention and minimising the risk of fluorosis'.

\section{Question 2: Is a DRV for 'habitual' or 'chronic intake', rather than acute toxicity?}

This question was prompted by a potential concern by some about the probable ingestion of products containing high concentrations of fluoride, such as varnishes, which were applied infrequently ( 2 to 4 applications per year) to the teeth of children and adults. It was noted that the amount of fluoride ingested ( $\mathrm{mgF} / \mathrm{kgbw} / \mathrm{day})$ following such applications was likely to be higher than current fluoride intake guidelines. The panel responded that a DRV refers to habitual intake. Infrequent ingestion of 
higher amounts of fluoride, as in the case of application of fluoride varnish, would be considered under toxicity, where a toxic level is many times usual intake.

\section{Question 3. Is there a need for an 'Upper limit (UL)' and/or 'Adequate intake (AI)' and/or any other DRV?}

All the reviewers had explained that high levels of fluoride intake can result in undesirable dental fluorosis, in the first instance. There was agreement that the modern trend of multiple sources of fluoride can result in fluoride ingestion close to this threshold, and the panel concluded that a ' $U L$ ' is definitely needed. However, there was discussion around the relevance of 'AI', and its definition, among the panel members. It was stressed that AI should not be confused with 'optimal' intake. This terminology has been examined and defined very similarly across several international reports such as the US Institute of Medicine (IoM), the European Food and Safety Authority (EFSA) and the Australian National Health and Medical Research Council (NHMRC). The discussion concluded with the statement that it is appropriate to have both 'UL' and 'AI' values as guidelines to influence public health policy when adjusting fluoride intake; an adequate intake to prevent dental caries without exceeding the intake levels that are linked with moderate to severe dental fluorosis. Since fluoride is considered as an important and beneficial element, but not yet an essential one, the panel felt that no other DRV terms are relevant for fluoride.

\section{Question 4: How should a UL for fluoride be established? Should it vary by age?}

As part of his review and presentation Dr. Spencer argued: "The RfD (equivalent to the UL) for the US EPA was estimated as the fluoride intake associated with the median drinking water consumption at the BMDL of $1.9 \mathrm{mg} \mathrm{F/L}$. Yet, no child in a population drinking water with $1.9 \mathrm{mg} F / L$ had the occurrence of severe fluorosis, the chosen end-point. Therefore, when calculating the fluoride intake at the BMDL the 95th percentile of drinking water consumption could be used. The UL will effectively double." This statement was discussed and the panel was in complete agreement with choosing the $95^{\text {th }}$ percentile of drinking water consumption for the calculation, as proposed by Dr Spencer. It was recognised that this was a significant recommendation.

In addition, it was pointed out that any recommendation on fluoride intake should take into account the degree of its absorption and bioavailability from different sources in line with similar estimations for any other nutrient, as recommended by the US Committee on Dietary Allowances (US National Research Council 1989): "The Food and Nutrition Board subcommittee exercised judgment in adjusting this value (i.e. RDA) by factors to compensate for incomplete utilization and to encompass the variation both in the requirements among individuals and in the bioavailability among the food sources of the nutrient." At this point, there was agreement with several of the reviewers that the 
bioavailability of fluoride differs depending on its source. For example, the bioavailability of fluoride from water is almost $100 \%$, whereas fluoride bioavailability from fluoridated milk is $72 \%$, from infant milk formula reconstituted with water it is 65\% (Spak et al. 1982) and in general a mixed diet may reduce absorption of fluoride by 47\% (Ekstrand and Ehrnebo 1979; Shulman and Vallejo 1990). It was noted that $\mathrm{AI}$ and $\mathrm{UL}$ values originated from when drinking water, with $100 \%$ fluoride bioavailability, was the main source ( $>70 \%$ ) of fluoride intake in children (MacClure 1943). However, in the present day of multiple sources of fluoride, the AI and UL values may be underestimated if the contribution of diet (with a lower degree of fluoride absorption) to total fluoride intake is taken into account, backing up the argument of a need to increase the threshold of UL.

It was recognised that age should be allowed for when setting up AI and UL. Further discussion concerned two specific age groups: infants of less than 6 months of age and the elderly. The first 6-8 years of life has been generally accepted as the window of susceptibility for possible, aestheticallyimportant fluorosis development in the permanent dentition. However, the panel argued that fluoride exposure during the first 6 months of life might carry less risk as at this age the formation of enamel may not be sufficiently advanced for fluorosis to develop.

The panel discussed whether the value of a UL for the elderly, which refers to the highest average daily nutrient intake level likely to pose no adverse health effects, had not been considered sufficiently. It was argued that development of dental fluorosis is irrelevant for this age group, but some key questions had yet to be addressed. What are the proven adverse effects for adults and in particular the elderly? Skeletal fluorosis, osteoporosis, hip fracture? What are the threshold values for such adverse effects? What is the balance between benefits and adverse effects in the elderly? Considering an average life expectancy of less than two and a half years for care home residents, should we not focus on maintaining oral health rather than concentrating on adverse effects? The optimum concentrations of fluoride for the prevention of root caries was considered to be higher than for the prevention of enamel caries. Evidence from studies in Hong Kong, concerning prevention of root caries in the elderly using high doses of topical applications of fluoride such as 'silver diamine fluoride (SDF)', was cited. The question was raised of whether the value of UL should be doubled for the elderly to prevent root caries. No conclusion was reached but it was recognised that the issue should be considered further.

\section{Question 5. Is there a need to measure fluoride intake? If so, should it be based on systemic intake and/or systemic absorption?}

The panel agreed on the need for assessment of fluoride intake to ensure that DRVs for fluoride are met at the population level. The panel disregarded any notion of trying to estimate absorption at the population level for monitoring DRVs. The panel agreed that there is not enough evidence to use 
biomarkers of fluoride exposure such as urine and nails, despite the fact that they take into account the absorption rate. The panel suggested that more research is needed to establish a reliable biomarker of fluoride exposure. In the meantime, direct measurement of fluoride intake from all sources (mainly diet and toothpaste ingestion) is therefore the most appropriate method for monitoring at the population level. The panel suggested including the rate of absorption into the risk model in scientific studies looking at the influence of fluoride intake on the risk of fluorosis.

Question 6: Should measurement of fluoride intake be part of national nutrition surveys? If so, should fluoride be included in food composition databases?

The panel agreed on the need to include measurement of fluoride intake in national diet and nutrition surveys. However, the potential major contribution of the use of fluoride-containing toothpastes to total fluoride intake, in children in particular, in addition to diet, was also discussed. The methods of assessment of fluoride intake from diet and ingestion of toothpaste were briefly discussed and it was considered that fluoride intake from toothpaste ingestion has been poorly investigated. Having national fluoride databases was considered desirable. Comprehensive fluoride databases are available in the UK and the US, whilst work is currently underway in Mexico and Columbia.

\section{Question 7: Is there a need for further research to improve/refine DRVs for fluoride? If so, should dental fluorosis be the driver for refining DRVs?}

This question was linked to two further questions: Can there be a consensus on the prevalence/severity of dental fluorosis which is unacceptable at the population level? This issue had been raised by virtually all speakers. Would a global symposium lead to any worthwhile clarity on this issue?

The meeting agreed that there is a need for further research and consideration to refine DRVs for fluoride. It was considered that biological (including genetic) factors, playing a role in both caries and fluorosis susceptibility, should be studied further. The field of fluoride metabolism, focusing on overall fluoride bioavailability/absorption and fluoride biomarkers combined with clinical studies, merits new research initiatives to provide more evidence-based data to inform DRVs for fluoride. The group considered that programmes for monitoring both caries and fluorosis should be established in all countries, focusing on collecting reliable data. Moreover, some sort of agreement should be reached on the type of indices to be used to measure the prevalence/severity of fluorosis due to its importance. It was concluded that these topics could be subjects for another symposium. 


\section{References:}

Ekstrand J, Ehrnebo M. 1979. Influence of milk products on fluoride bioavailability in man. Eur J Clin Pharmacol. 16(3):211-215.

McClure FJ. 1943. Ingestion of fluoride and dental caries. Quantitative relations based on food and water requirements of children 1-12 years old. Am J Dis Child. 66(4): 362-369.

Nielsen FH. 1996. How should dietary guidance be given for mineral elements with beneficial actions or suspected of being essential? J Nutr. 126(9):S2377-S2385.

Shulman ER, Vallejo M. 1990. Effect of gastric contents on the bioavailability of fluoride in humans. Pediatr Dent. 12(4):237-240.

Spak CJ, Ekstrand J, Zylberstein D. 1982. Bioavailability of fluoride added to baby formula and milk. Caries Res. 16:249-256.

US National Research Council. 1989. Recommended dietary allowance. Washington (USA): National Academy of Sciences. 
Declaration of interests

The author reports no conflicts of interest. 